\title{
QUIVER VARIETIES, AFFINE LIE ALGEBRAS, ALGEBRAS OF BPS STATES, AND SEMICANONICAL BASIS
}

\author{
IGOR FRENKEL, ANTON MALKIN, AND MAXIM VYBORNOV
}

\begin{abstract}
We suggest a (conjectural) construction of a basis in the plus part of the affine Lie algebra of type ADE indexed by irreducible components of certain quiver varieties. This construction is closely related to a stringtheoretic construction of a Lie algebra of BPS states. We then study the new combinatorial questions about the (classical) root systems naturally arising from our constructions and Lusztig's semicanonical basis.
\end{abstract}

\section{IntRoduCtion}

1.1. Since the appearance of the first manifestation of the connection between theory of representations of quivers and the structure theory of Lie algebras Gab72, several authors discovered constructions of Lie algebras arising from the quiver theory. C. M. Ringel constructed the plus part of the simple Lie algebra of type ADE, Rin90], and a (related) construction of the plus part of any Kac-Moody Lie algebra is implicit in Lusztig's construction of (quantized) enveloping algebras of Kac-Moody Lie algebras in terms of functions on a class of remarkable affine varieties $\Lambda_{V}$, Lus91, Lus92].

Inspired by Ringel and Lusztig, the present authors came up with a construction of the plus part $\widetilde{\mathfrak{n}}_{+}$of the affine Lie algebra of type ADE in terms of indecomposable representations of quivers [FMV01.

A similar construction is suggested by string theorists FM00, who use the stable representations of (double) quivers rather than indecomposable representations of oriented quivers (as do Ringel and the present authors). The concept of stability has been very useful in representation theory: H. Nakajima discovered that modules over Kac-Moody Lie algebras may be described using functions on the varieties $\Lambda_{V}^{s} / G_{V}$ where $\Lambda_{V}^{s}$ are the stable points of Lusztig's varieties $\Lambda_{V}$ [Nak94, Nak98].

1.2. One of the main goals of this paper is to study the relationship between the Lusztig's construction of $\tilde{\mathfrak{n}}_{+}$, the construction by the present authors [FMV01, and the "stable" construction suggested by physicists. Using the notion of semistable diagonal in $\Lambda_{V}$ suggested in HM98, we make a conjecture (Conjecture 4.2.5) directly relating the Lusztig's construction and the "stable" construction. Moreover, we (conjecturally) obtain a basis in $\widetilde{\mathfrak{n}}_{+}$parameterized by irreducible components of algebraic varieties. One can look at this conjectural basis as "semicanonical" basis for $\widetilde{\mathfrak{n}}_{+}$.

1.3. In section 5 we use our methods to obtain some simple results describing new aspects (arising from quiver constructions) of combinatorics of the root systems and Weyl groups, and ask many more questions than we can answer at the moment. 
One of the results of the Lusztig construction of the (quantized) universal enveloping algebras in terms of functions on the varieties $\Lambda_{V}$ is the appearance of the semicanonical basis in the non-quantized enveloping algebras of simply laced Kac-Moody Lie algebras Lus00]. Semicanonical basis is indexed by the irreducible components of $\Lambda_{V}$. It was expected that this basis would coincide with the specialization of the Lusztig's canonical basis to $q=1$, but a counterexample was found by M. Kashiwara and Y. Saito KS97. The relationship between these two bases is quite mysterious at the moment, even though they share their combinatorial properties.

Let $\mathfrak{n}_{+} \subset U\left(\mathfrak{n}_{+}\right)$be the plus part of a simple Lie algebra of type ADE, and $U\left(\mathfrak{n}_{+}\right)$be its universal enveloping algebra. For every positive root $\alpha \in R_{+}$we have a canonically (up to a sign) defined element $E_{\alpha}^{*} \in \mathfrak{n}_{\alpha} \subset U\left(\mathfrak{n}_{+}\right)$, where $\mathfrak{n}_{\alpha}$ is the one dimensional root subspace of $\mathfrak{n}_{+}$corresponding to $\alpha$. We study the decomposition of $E_{\alpha}^{*}$ with respect to the semicanonical basis. More precisely, let us consider $\Lambda_{V}, \operatorname{dim} V=\alpha$. Then the irreducible components of $\Lambda_{V}$ may be indexed by decompositions of $\alpha$ into the sum of positive roots. Let $\alpha=\alpha_{1}+\alpha_{2}+\cdots+\alpha_{l}$, $\alpha_{i} \in R_{+}$be such a decomposition and let $e_{\alpha_{1}+\alpha_{2}+\cdots+\alpha_{l}}$ be the element of the semicanonical basis corresponding to this decomposition (and the corresponding irreducible component of $\Lambda_{V}$ ). Then

$$
E_{\alpha}^{*}=\sum_{\alpha=\alpha_{1}+\alpha_{2}+\cdots+\alpha_{l}} c_{\alpha_{1}+\alpha_{2}+\cdots+\alpha_{l}} e_{\alpha_{1}+\alpha_{2}+\cdots+\alpha_{l}}
$$

where $c_{\alpha_{1}+\alpha_{2}+\cdots+\alpha_{l}} \in \mathbb{Z}$ is the coefficient of $e_{\alpha_{1}+\alpha_{2}+\cdots+\alpha_{l}}$. In other words, with the help of the semicanonical basis we assign an integer $c_{\alpha_{1}+\alpha_{2}+\cdots+\alpha_{l}}$ to every decomposition $\alpha=\alpha_{1}+\alpha_{2}+\cdots+\alpha_{l}$ of the root $\alpha$ into a sum of positive roots. It would be very interesting give a purely combinatorial description of these numbers without appealing to the semicanonical basis. We manage to obtain such a description in the $A_{n}$ case in terms of the sign character of the Weyl group $W=S_{n+1}$.

1.4. Acknowledgment. We are grateful to Naihuan Jing who presented us with the opportunity to give this talk at the CBMS conference he organized at NCSU in June 2001. We tried to preserve a bit of the informal style of a talk in this paper. We are grateful to B. Fiol, J. Humphreys, G. Lusztig, M. Mariño, and C. Ringel for useful conversations. M.V. is grateful to MSRI and Max-Planck-Institut für Mathematik, where parts of this work were done, for their hospitality. The research of I. F. was supported in part by NSF. The research of A. M. and M. V. was supported by NSF Postdoctoral Research Fellowships.

\section{Preliminaries}

We always assume that our ground field is the field of complex numbers $\mathbb{C}$. Our notation and conventions are mostly lifted from LLus91, Lus00, FMV01.

\subsection{Quivers.}

2.1.1. To a graph $Q$, with no edges joining a vertex with itself, we associate a pair of sets: $I$ (vertices), and $H$ (oriented edges), and two maps from $H$ to $I$ :

2.1.1.a. a map $H \rightarrow I$ denoted $h \rightarrow h^{\prime}$ (initial vertex),

2.1.1.b. a map $H \rightarrow I$ denoted $h \rightarrow h^{\prime \prime}$ (terminal vertex),

2.1.1.c. an involution $h \rightarrow \bar{h}$ on $H$ which maps an oriented edge to the same edge with the opposite orientation. 
An orientation of $(I, H)$ is a choice of a subset $\Omega \subset H$ such that $\Omega \cup \bar{\Omega}=H$ and $\Omega \cap \bar{\Omega}=\emptyset$. Abusing terminology we will call both $(I, H)$ and $(I, \Omega)$ quivers.

2.1.2. Let $\mathcal{V}$ be the category of $I$-graded vector spaces $V=\oplus_{i \in I} V_{i}$. We define:

$$
\begin{aligned}
\mathbf{E}_{V} & =\bigoplus_{h \in H} \operatorname{Hom}\left(V_{h^{\prime}}, V_{h^{\prime \prime}}\right), \\
\mathbf{E}_{V, \Omega} & =\bigoplus_{h \in \Omega} \operatorname{Hom}\left(V_{h^{\prime}}, V_{h^{\prime \prime}}\right) .
\end{aligned}
$$

For an element $x \in \mathbf{E}_{V}$ we denote by $x_{h}, h \in H$ its component in $\operatorname{Hom}\left(V_{h^{\prime}}, V_{h^{\prime \prime}}\right)$. A pair $(V, x), x \in \mathbf{E}_{V}$ (resp. $x \in \mathbf{E}_{V, \Omega}$ ) is called a representation of $(I, H)$ (resp. $(I, \Omega))$. We will also sometimes call $(V, x)$ a module over $(I, H)$, and moreover denote such a module by $V$ if it is clear what $x$ we consider.

Let $\mathbb{Z}[I]$ be the free abelian group generated by the set $I$. The dimension of $(V, x)$ with $x \in \mathbf{E}_{V}$ or $x \in \mathbf{E}_{V, \Omega}$ is an element of $\mathbb{Z}[I]$ defined as follows:

$$
\operatorname{dim}(V, x)=\operatorname{dim} V=\sum_{i \in I} \operatorname{dim}_{\mathbb{C}}\left(V_{i}\right) i \in \mathbb{Z}[I] .
$$

The algebraic group $G_{V}=\prod_{i \in I} G L\left(V_{i}\right)$ acts on $\mathbf{E}_{V}$ in a natural way.

2.1.3. We say that $x \in \mathbf{E}_{V, \Omega}$ is indecomposable if $(V, x)$ is indecomposable as a representation of $(I, \Omega)$. The subset of indecomposable elements in $\mathbf{E}_{V, \Omega}$ is denoted by $\mathbf{E}_{V, \Omega}^{\text {ind }}$.

2.1.4. We say that $x \in \mathbf{E}_{V}$ is nilpotent if there exists an $N \geq 2$ such that for any sequence $h_{1}, h_{2}, \ldots, h_{N} \in H$, such that $h_{1}^{\prime}=h_{2}^{\prime \prime}, h_{2}^{\prime}=h_{3}^{\prime \prime}, \ldots, h_{N-1}^{\prime}=h_{N}^{\prime \prime}$, the composition $x_{h_{1}} x_{h_{2}} \ldots x_{h_{N}}=0$. The subset of nilpotent elements in $\mathbf{E}_{V}$ is denoted by $\mathbf{E}_{V}^{n i l}$.

2.1.5. Following Lus91] we consider the moment map attached to the $G_{V^{-}}$-action on $\mathbf{E}_{V}$. The $i$-component $m_{i}$ of this map is given by

$$
m_{i}(x)=\sum_{h \in H: h^{\prime \prime}=i} \epsilon(h) x_{h} x_{\bar{h}},
$$

where $\epsilon: H \rightarrow \mathbb{C}^{*}$ is a function such that $\epsilon(h)+\epsilon(\bar{h})=0$ for all $h \in H$. Following Lusztig we introduce the subvariety $\Lambda_{V}$ of $\mathbf{E}_{V}$ as follows

Definition. $\Lambda_{V}$ is the closed subvariety of all nilpotent elements $x \in \mathbf{E}_{V}$ such that $m_{i}(x)=0$ for all $i \in I$.

\subsection{Convolution product.}

2.2.1. Let

$$
\mathcal{L}(V)=M_{G_{V}}\left(\Lambda_{V}\right)
$$

be the set of constructible $\mathbb{C}$-valued functions on $\Lambda_{V}$ which are constant on $G_{V^{-}}$ orbits in $\Lambda_{V}$. Let $\mathcal{L}_{\mathbb{Q}}(V)$ (resp. $\left.\mathcal{L}_{\mathbb{Z}}(V)\right)$ be the set of all $f \in \mathcal{L}(V)$ with rational (resp. integral) values. 
2.2.2. Let $V, V^{\prime}, V^{\prime \prime} \in \mathcal{V}$ be such that $\operatorname{dim} V^{\prime}+\operatorname{dim} V^{\prime \prime}=\operatorname{dim} V$. Let $f^{\prime} \in \mathcal{L}\left(V^{\prime}\right)$, $f^{\prime \prime} \in \mathcal{L}\left(V^{\prime \prime}\right)$. We lift the convolution construction from [Lus91, 12.10]. Consider the diagram

$$
\Lambda_{V^{\prime}} \times \Lambda_{V^{\prime \prime}} \stackrel{p_{1}}{\longleftarrow} \mathbf{F}^{\prime} \stackrel{p_{2}}{\longrightarrow} \mathbf{F}^{\prime \prime} \stackrel{p_{3}}{\longrightarrow} \Lambda_{V}
$$

where $\mathbf{F}^{\prime \prime}$ is the variety of all pairs $(x, W)$ where $x \in \Lambda_{V}$ and $W \subset V$ is an $x$-stable subspace, $\operatorname{dim} W=\operatorname{dim} V^{\prime \prime}$.

$\mathbf{F}^{\prime}$ is the variety of all quadruples $\left(x, W, R^{\prime \prime}, R^{\prime}\right)$ where $(x, W) \in \mathbf{F}^{\prime \prime}$, and $R^{\prime}, R^{\prime \prime}$ are $\mathcal{V}$-isomorphisms $R^{\prime}: V^{\prime} \simeq V / W, R^{\prime \prime}: V^{\prime \prime} \simeq W$.

We have $p_{1}\left(x, W, R^{\prime \prime}, R^{\prime}\right)=\left(x^{\prime}, x^{\prime \prime}\right)$ where $x_{h} R_{h^{\prime}}^{\prime}=R_{h^{\prime \prime}}^{\prime} x_{h}^{\prime}$, and $x_{h} R_{h^{\prime}}^{\prime \prime}=R_{h^{\prime \prime}}^{\prime \prime} x_{h}^{\prime \prime}$ for all $h \in H$.

We have $p_{2}\left(x, W, R^{\prime \prime}, R^{\prime}\right)=(x, W), p_{3}(x, W)=x$.

Let $f_{1} \in \mathcal{L}\left(\Lambda_{V^{\prime}} \times \Lambda_{V^{\prime \prime}}\right)$ be given by $f_{1}\left(x^{\prime}, x^{\prime \prime}\right)=f^{\prime}\left(x^{\prime}\right) f^{\prime \prime}\left(x^{\prime \prime}\right)$. Then there is a unique function $f_{3} \in \mathcal{L}\left(\mathbf{F}^{\prime \prime}\right)$ such that $p_{1}^{*} f_{1}=p_{2}^{*} f_{3}$. Then by definition

$$
f^{\prime} * f^{\prime \prime}=\left(p_{3}\right) !\left(f_{3}\right)
$$

There exists an analogous construction for the oriented quiver [Lus91, 10.19]. We will denote the corresponding convolution product by $*_{\Omega}$ for an orientation $\Omega$.

2.2.3. If $\operatorname{dim} V=i \in I$, then $\Lambda_{V}$ is a point, and we denote by $E_{i}\left(\Lambda_{V}\right) \equiv 1$ the function which is identically 1 on this point. Let $\mathcal{L}_{0}$ be the associative algebra with *-product generated by $\left\{E_{i}\right\}_{i \in I}$. (The associative algebra of functions on $\mathbf{E}_{V, \Omega}$ with $* \Omega$-product generated by $\left\{E_{i}\right\}_{i \in I}$ will be denoted by $\mathcal{L}_{0, \Omega}$.) One can consider $\mathcal{L}_{0}$ as a $\mathbb{Z}_{+}[I]$-graded Lie algebra over $\mathbb{Q}$, with the following Lie bracket:

$$
[f, g]=f * g-g * f .
$$

We denote by $\mathfrak{n}^{*}$ the Lie subalgebra of $\mathcal{L}_{0}$ generated by $\left\{E_{i}\right\}_{i \in I}$.

\subsection{Geometric realization of the enveloping algebra.}

2.3.1. Let $(I, H)$ be a quiver. For $i, j \in I$ define $i \cdot j=-\operatorname{Card}\left\{h \in H \mid h^{\prime}=\right.$ $i, h^{\prime \prime}=j$, if $i \neq j$ and $i \cdot j=2$ if $i=j$. Let $U^{+}$be the $\mathbb{C}$-algebra defined by generators $e_{i}, i \in I$ and the Serre relations:

$$
\sum_{\substack{p, q \in \mathbb{Z}_{\geq 0} \\ p+q=-i \cdot j+1}}(-1)^{p} \frac{e_{i}^{p}}{p !} e_{j} \frac{e_{i}^{q}}{q !}=0
$$

for any $i \neq j$ in $I . U^{+}=U(\mathfrak{n})$ is the enveloping algebra of the Lie algebra $\mathfrak{n}$ defined by generators $e_{i}, i \in I$ and the Serre relations:

$$
\left(\operatorname{ad} e_{i}\right)^{-i \cdot j+1}\left(e_{j}\right)=0
$$

for any $i \neq j$ in $I$.

Let $(V, x)$ be a representation of $(I, H)$. Let $U_{\operatorname{dim} V}^{+}$denote the subspace of $U^{+}$ generated by $e_{i_{1}} e_{i_{2}} \ldots e_{i_{n}}$ for sequences $i_{1}, i_{2}, \ldots, i_{n}$ in which $i$ appears $\operatorname{dim} V_{i}$ times for any $i \in I$.

Let $U_{\mathbb{Z}}^{+}$be the subring of $U^{+}$generated by the elements $e_{i}^{p} / p$ ! for all $i \in I$, $p \in \mathbb{Z}_{\geq 0}$. Then $U_{\mathbb{Z}, \operatorname{dim} V}^{+}=U_{\mathbb{Z}}^{+} \cap U_{\operatorname{dim} V}^{+}$, see Lus00, 1.1]. 
2.3.2. In Lus91, 12.12] Lusztig defines a $\mathbb{C}$-linear map $\psi_{V}: U_{\operatorname{dim} V}^{+} \rightarrow \mathcal{L}(V)$, such that

2.3.2.a. $\psi=\bigoplus_{\operatorname{dim} V} \psi_{V}: U^{+} \simeq \mathcal{L}_{0}$ is an isomorphism of algebras,

2.3.2.b. $\psi\left(e_{i}\right)=E_{i}$, for any $i \in I$,

2.3.2.c. $\psi_{V}\left(U_{\mathbb{Z}, \operatorname{dim} V}^{+}\right) \subset \mathcal{L}_{\mathbb{Z}}(V)$.

The isomorphism $\psi$ also restricts to an isomorphism of Lie algebras $\psi: \mathfrak{n} \rightarrow \mathfrak{n}^{*}$ such that $\psi\left(e_{i}\right)=E_{i}$ for any $i \in I$.

In Lus00 Lusztig defines a $\mathbb{Q}$ version of the map $\psi_{V}: U_{\operatorname{dim} V}^{+} \rightarrow \mathcal{L}(V)$, which restricts to a map $\psi_{V}: U_{\mathbb{Z}, \operatorname{dim} V}^{+} \rightarrow \mathcal{L}_{\mathbb{Z}}(V)$.

2.4. Lie algebra based on the Euler cocycle: classical ADE case. Let the graph underlying the quiver $(I, H)$ be the Dynkin diagram of type ADE. In this case we can identify $\mathbb{Z}[I]$ with the root lattice of type ADE. The elements $i \in I$ are considered simple roots, and we have the root system $R \subset \mathbb{Z}[I]$ and its positive part $R_{+} \subset \mathbb{Z}_{\geq 0}[I]$.

Let us fix a cocylcle $\epsilon: \mathbb{Z}[I] \times \mathbb{Z}[I] \rightarrow \mathbb{Z} / 2 \mathbb{Z}$. In the simply laced Dynkin diagram case, the Lie algebra $\mathfrak{n}=\mathfrak{n}^{*}$ defined above is isomorphic to the Lie algebra $n^{\epsilon}$ spanned by the elements $\tilde{e}_{\alpha}, \alpha \in R_{+}$with the bracket defined by $\epsilon$ as in [FMV01, 4.2.1.a].

Now let us define the functions $E_{\alpha}^{*} \in \mathcal{L}(V), \alpha \in R_{+}, \operatorname{dim} V=\alpha$ as follows:

$$
E_{\alpha}^{*}=\psi\left(\tilde{e}_{\alpha}\right) \text {. }
$$

Notice that if $\alpha=i \in I$, then $\Lambda_{V}$ is a point and $E_{i}^{*}=E_{i}$

2.5. Lie algebra based on the Euler cocycle: affine ADE case. Let the graph underlying the quiver $(I, H)$ be the extended Dynkin diagram of type ADE. In this case we can identify $\mathbb{Z}[I]$ with the affine root lattice of type ADE. The elements $i \in I$ are considered simple roots, and we have the affine root system $R \subset \mathbb{Z}[I]$ and its positive part $R_{+} \subset \mathbb{Z}_{\geq 0}[I]$.

Let us choose an extending vertex $p \in I$, and let $I^{\prime}=I-\{p\}$.

Again, let us fix a cocylcle $\epsilon: \mathbb{Z}[I] \times \mathbb{Z}[I] \rightarrow \mathbb{Z} / 2 \mathbb{Z}$. Let $\mathfrak{n}_{\alpha}$ be the root space of the algebra $\mathfrak{n}$ corresponding to $\alpha \in R_{+}$. Let $\delta$ be the indivisible imaginary root. The Lie algebra $\mathfrak{n}$ is isomorphic to the Lie algebra $\mathfrak{n}^{\epsilon}$ spanned by the elements $\tilde{e}_{\alpha} \in \mathfrak{n}_{\alpha}^{\epsilon}, \alpha \in R_{+}^{\mathrm{re}}$, and $\alpha_{k}(n) \in \mathfrak{n}_{n \delta}^{\epsilon}, n \geq 1$, where we identify $\mathfrak{n}_{n \delta}^{\epsilon}=\mathbb{C}\left[I^{\prime}\right]=$ the vector space spanned by $I^{\prime}$, and $\alpha_{k}=k \in I^{\prime} \subset \mathbb{Z}\left[I^{\prime}\right]$ are simple roots. The bracket is defined by $\epsilon$ as in [FMV01, 5.2.1.a]. In particular,

$$
\left[\tilde{e}_{\alpha}, \tilde{e}_{\beta}\right]= \begin{cases}\epsilon(\alpha, \beta) \tilde{e}_{\alpha+\beta} & \text { if } \alpha+\beta \in R_{+}^{\mathrm{re}}, \\ \epsilon(\alpha, \beta) \alpha(n) & \text { if } \alpha+\beta=n \delta, \\ 0 & \text { if } \alpha+\beta \notin R_{+} .\end{cases}
$$

Now let us define the functions $E_{\alpha}^{*} \in \mathcal{L}(V), \operatorname{dim} V=\alpha \in R_{+}^{\text {re }}$ as follows:

$$
E_{\alpha}^{*}=\psi\left(\tilde{e}_{\alpha}\right),
$$

and the functions $E_{k}^{*}(n) \in \mathcal{L}(V), \operatorname{dim} V=n \delta$ as follows:

$$
E_{k}^{*}(n)=\psi\left(\alpha_{k}(n)\right)
$$

Again, if $\alpha=i \in I$, then $\Lambda_{V}$ is a point and $E_{i}^{*}=E_{i}$. 


\section{Stability and simple Lie algebras of type ADE}

\subsection{Stability after King and Rudakov.}

3.1.1. Stability after King. Let us consider the abelian category of nilpotent representations of a quiver $(I, H)$ and its Grothendieck group $K_{0}$. A character on $K_{0}$ is an additive function $\theta: K_{0} \rightarrow \mathbb{R}$.

Definition. King94 A point $x \in \mathbf{E}_{V}$ is called $\theta$-stable (resp. $\theta$-semistable) if $\theta(V)=0$, and for any $x$-stable nonzero proper subspace $V^{\prime} \subset V$ we have $\theta\left(V^{\prime}\right)>0$ (resp. $\left.\theta\left(V^{\prime}\right) \geq 0\right)$.

Definition. Two $\theta$-semistable points $x^{\prime}, x^{\prime \prime} \in \mathbf{E}_{V}$ are $S$-equivalent (notation: $x^{\prime} \stackrel{S}{\sim}$ $\left.x^{\prime \prime}\right)$ if the orbit closures $\overline{G_{V} \cdot x^{\prime}} \cap \overline{G_{V} \cdot x^{\prime \prime}}$ intersect in the set of $\theta$-semistable points $\mathbf{E}_{V}^{s s}$.

3.1.2. Stability after Rudakov. One can also define stable points using a "slope" stability condition: $\mu=c / r$ where $c$ and $r$ are additive functions $K_{0} \rightarrow \mathbb{R}$ and $r(V)>0$ for any $V \in \mathcal{V}$, see [Rud97, 3].

Definition. Rud97 A point $x \in \mathbf{E}_{V}$ is called $\mu$-stable (resp. $\mu$-semistable) if for any $x$-stable nonzero proper subspace $V^{\prime} \subset V$ we have $\mu\left(V^{\prime}\right)<\mu(V)$ (resp. $\left.\mu\left(V^{\prime}\right) \leq \mu(V)\right)$.

If we fix such slope stability condition $\mu$ and $V \in \mathcal{V}$ we can define a character $\theta_{\mu}: K_{0} \rightarrow \mathbb{R}$ as follows:

$$
\theta_{\mu}\left(V^{\prime}\right)=-c\left(V^{\prime}\right)+\frac{c(V)}{r(V)} r\left(V^{\prime}\right)
$$

According to [Rud97, Proposition 3.4] a point $x \in \mathbf{E}_{V}$ is $\mu$-stable if and only if it is $\theta_{\mu}$-stable.

3.1.3. Let us fix an orientation $\Omega \subset H$ until the end of this section. Following King94 we construct a character $\theta=\Theta_{V, \Omega}$ associated to $\Omega$. Let $V, V^{\prime} \in \mathcal{V}$. We define

$$
\Theta_{V, \Omega}\left(V^{\prime}\right)=\sum_{h \in \Omega}\left(\operatorname{dim} V_{h^{\prime}} \operatorname{dim} V_{h^{\prime \prime}}^{\prime}-\operatorname{dim} V_{h^{\prime}}^{\prime} \operatorname{dim} V_{h^{\prime \prime}}\right) .
$$

3.2. Stability Lemma. In this section we will consider quivers of finite ADE type, i.e. the underlying non-oriented graph $Q$ is a Dynkin graph of (finite) ADE type. A fact similar to the lemma below was independently conjectured by M. Reineke Rei02, Conjecture 7.1]

Lemma. Let $x \in \Lambda_{V}, \operatorname{dim} V=\alpha \in R_{+}$. Then $x$ is $\Theta_{V, \Omega}$-stable if and only if $x \in \mathbf{E}_{V, \Omega}^{\text {ind }}$.

Proof. Since in the Dynkin quiver case every root is a Schur root King94, a generic point in $\mathbf{E}_{V, \Omega}$ is $\Theta_{V, \Omega}$-stable according to [King94, Scho92]. Since $\mathbf{E}_{V, \Omega}^{\text {ind }}$ is an open dense $G_{V}$-orbit in $\mathbf{E}_{V, \Omega}$, any $x \in \mathbf{E}_{V, \Omega}^{\text {ind }}$ is $\Theta_{V, \Omega}$-stable.

Let us prove the "only if" part. If $x \in \Lambda_{V}-\mathbf{E}_{V, \Omega}^{\text {ind }}$, then we have $x=y+z$, where $y \in \mathbf{E}_{V, \Omega}, z \in \mathbf{E}_{V, \bar{\Omega}}$. Following [Lus91, 14] we see that there exists a decomposition:

$$
(V, y)=\oplus_{p=1}^{\nu}\left(V^{p}, y\right)
$$


such that $\operatorname{Hom}\left(V^{p}, V^{p^{\prime}}\right)=0$ whenever $p^{\prime}<p$. There is a direct sum decomposition:

$$
\mathbf{E}_{V, \Omega}=\bigoplus_{1 \leq p, p^{\prime} \leq \nu} \mathbf{E}_{V, \Omega}^{p, p^{\prime}} \quad \text { where } \quad \mathbf{E}_{V, \Omega}^{p, p^{\prime}}=\operatorname{Hom}_{h \in \Omega}\left(V_{h^{\prime}}^{p}, V_{h^{\prime \prime}}^{p^{\prime}}\right) .
$$

Now we need the following claim: there exists $y^{\prime} \in \bigoplus_{p>p^{\prime}} \mathbf{E}_{V, \Omega}^{p, p^{\prime}}$ such that $y+y^{\prime}=$ : $x^{\prime} \in \mathbf{E}_{V, \Omega}^{\text {ind }}$. It is not very hard to prove this claim using the methods of [Lus90, 4].

Following [Lus91, 14] we denote $V^{(q)}=\oplus_{p>q} V^{p}$. Then we have the $x$-invariant filtration:

$$
V=V^{(0)} \supset V^{(1)} \supset \cdots \supset V^{(\nu)}=0 .
$$

Denote $V_{(q)}=\oplus_{p<q} V^{p}$. Then we have the $x^{\prime}$-invariant filtration:

$$
0=V_{(0)} \subset V_{(1)} \subset \cdots \subset V_{(\nu)}=V .
$$

The vector space $V_{(q)}$ is a submodule in $\left(V, x^{\prime}\right)$. Since $x^{\prime}$ is $\Theta_{V, \Omega}$-stable we have $\Theta_{V, \Omega}\left(V_{(q)}\right)>0$. However $V_{(q)}$ is a quotient module in $(V, x)$. Therefore, $(V, x)$ is unstable.

\subsection{Stable construction.}

3.3.1. Let us consider the cocycle $\epsilon_{\Omega}$ associated to our fixed orientation $\Omega$, see FK81], FMV01, 1.3.4]. By construction, the functions $E_{\alpha}^{*}, \alpha \in R_{+}$on $\Lambda_{V}$ defined as in 2.4 with the cocycle $\epsilon=\epsilon_{\Omega}$ form a basis of the Lie algebra $n^{*}$, and the *-bracket is given by:

$$
\left[E_{\alpha}^{*}, E_{\beta}^{*}\right]= \begin{cases}\epsilon(\alpha, \beta) E_{\alpha+\beta}^{*}, & \text { if } \alpha+\beta \in R_{+}, \\ 0 & \text { if } \alpha+\beta \notin R_{+} .\end{cases}
$$

3.3.2. It is clear that the affine variety $\mathbf{E}_{V, \Omega} \subset \Lambda_{V}$ is one of the irreducible components of $\Lambda_{V}$. We can define a constructible function $E_{\alpha}, \alpha \in R_{+}$on $\mathbf{E}_{V, \Omega}$, $\operatorname{dim} V=\alpha$ as follows, see [FMV01, 4.3.1.b]:

$$
E_{\alpha}= \begin{cases}1, & \text { if } x \in \mathbf{E}_{V, \Omega}^{\text {ind }} \\ 0 & \text { otherwise }\end{cases}
$$

According to [FMV01, 4.3.4], the space spanned by the functions $E_{\alpha}, \alpha \in R_{+}$ is a Lie algebra (which we will denote here by $\mathfrak{n}^{\Omega}$ ) with the bracket $[f, g]_{\Omega}=$ $f *_{\Omega} g-g *_{\Omega} f$. The map $\mathfrak{n}^{\epsilon} \rightarrow \mathfrak{n}^{\Omega}$ given by $\tilde{e}_{\alpha} \rightarrow E_{\alpha}$ is an isomorphism. In other words, the bracket can be explicitly described as follows:

$$
\left[E_{\alpha}, E_{\beta}\right]_{\Omega}= \begin{cases}\epsilon(\alpha, \beta) E_{\alpha+\beta}, & \text { if } \alpha+\beta \in R_{+}, \\ 0 & \text { if } \alpha+\beta \notin R_{+} .\end{cases}
$$

3.3.3. Let us take the character $\Theta_{V, \Omega}$, and denote the identity function on $\Lambda_{V}^{s}$, $\operatorname{dim} V=\alpha$, by $\widetilde{E}_{\alpha}, \widetilde{E}_{\alpha}\left(\Lambda_{V}^{s}\right) \equiv 1$, where $\Lambda_{V}^{s}$ is the set of points in $\Lambda_{V}$ stable with respect to $\Theta_{V, \Omega}$. Notice that by Lemma 3.2, $\Lambda_{V}^{s}=\mathbf{E}_{V, \Omega}^{\text {ind }}$, and so the function $\widetilde{E}_{\alpha}$ is constant on a single $G_{V}$-orbit in $\Lambda_{V}^{s}$.

Lemma. Up to a sign:

3.3.3.a. $\left.E_{\alpha}^{*}\right|_{\mathbf{E}_{V, \Omega}}=E_{\alpha}$,

3.3.3.b. $\left.E_{\alpha}^{*}\right|_{\Lambda_{V}^{s}}=\widetilde{E}_{\alpha}$.

Proof. Follows immediately from definitions, the proof of [Lus91, Theorem 12.13], and the formulas 3.3.1.a, 3.3.2.b. 
3.3.4. Let us denote the space spanned by the functions $\widetilde{E}_{\alpha}, \alpha \in R_{+}$by $\mathfrak{n}^{\text {stable }}$. Since $\Lambda_{V}^{s}=\mathbf{E}_{V, \Omega}^{\text {ind }}$, by lemma 3.2, the restrictions from $\Lambda_{V}$ to $\mathbf{E}_{V, \Omega}$ to $\Lambda_{V}^{s}$ provide us with the based isomorphisms of vector spaces (cf. Lemma 3.3.3)

$$
\mathfrak{n}^{*} \underset{\text { restriction }}{\stackrel{\simeq}{\longrightarrow}} \mathfrak{n}^{\Omega} \underset{\text { restriction }}{\stackrel{\simeq}{\longrightarrow}} \mathfrak{n}^{\text {stable }}
$$

defined by $E_{\alpha}^{*} \mapsto E_{\alpha} \mapsto \widetilde{E}_{\alpha}$ for $\alpha \in R_{+}$. It is clear that the first restriction is an isomorphism of Lie algebras, and the second restriction equips the space $\mathfrak{n}^{\text {stable }}$ of functions on $\Lambda_{V}^{s}$ constant on $G_{V}$-orbits (or equivalently, functions on $\left.\Lambda_{V}^{s} / G_{V}=\mathrm{pt}_{V}\right)$ with the structure of a Lie algebra isomorphic to $\mathfrak{n}^{*} \simeq \mathfrak{n}^{\Omega} \simeq \mathfrak{n}^{\epsilon}$.

\section{Stability, Affine Lie algebras of type ADE, And a Conjectural CONSTRUCTION OF THE ALGEBRAS OF BPS STATES}

\subsection{Physics.}

4.1.1. One of the current models of string theory defines $D$-branes as objects in the derived category $D^{b}(\operatorname{Coh}(Z))$ of the coherent sheaves on the algebraic (Calabi-Yau) variety $Z$. Physicists consider the moduli space $\mathcal{M}_{\zeta}(n)$ (where $\zeta=\left(\zeta_{1}, \zeta_{2}, \ldots, \zeta_{N}\right)$ are the Fayet-Iliopulos terms) of semistable D-branes of charge $n$, cf. HM98, DFR00, FM00]. In the semiclassical approximation, the space of BPS states is given by the cohomology of the moduli space:

$$
\mathcal{H}_{\mathrm{BPS}}=H^{*}\left(\mathcal{M}_{\zeta}(n)\right)
$$

4.1.2. Dictionary. We will consider the $D$-branes on the resolution $\widetilde{\mathbb{C}^{2} / \Gamma}$ of a simple singularity $\mathbb{C}^{2} / \Gamma$. Let $Q$ be the extended Dynkin diagram corresponding to $\Gamma$ via the McKay correspondence. We suggest a mathematical model of the physical situation in this case with which we will work. In the next subsection 4.1.3 we will justify our model. For now, we provide the physics/mathematics dictionary:

the charge lattice : the positive part $\mathbb{Z}_{\geq 0}[I]$ of the affine root lattice associated to $Q$;

places in the the charge lattice occupied by the single particle BPS states: positive roots $R_{+} \subset \mathbb{Z}_{\geq 0}[I]$;

a D-brane of charge $\alpha \in \mathbb{Z}[I]$ on $\widetilde{\mathbb{C}^{2} / \Gamma}$ : a representation $x \in \Lambda_{V}, \operatorname{dim} V=\alpha$ of the quiver $(\mathrm{I}, \mathrm{H})$ (with the underlying non-oriented graph $Q$ );

Fayet-Iliopulos terms $\zeta$ : an additive function $c$ which defines Rudakov's slope stability together with $r(V)=\sum_{i \in I} \operatorname{dim} V_{i}$;

$\zeta$-stable $D$-branes : stable elements of $\Lambda_{V}$ with respect to a stability condition; moduli spaces of $D$-branes of charge $\alpha \in R_{+}^{\mathrm{re}}: \Lambda_{V}^{s} / G_{V}=\mathrm{pt}_{\alpha}, \operatorname{dim} V=\alpha \in R_{+}^{\mathrm{re}}$; moduli spaces of D-branes of charge $\delta=$ indivisible imaginary root: $\Lambda_{V}^{s} / G_{V}=$ $\mathfrak{L}=\mathfrak{L}_{1}$, where $\mathfrak{L}$ is the exceptional fiber of the resolution of simple singularity;

moduli spaces of D-branes of charge $m \delta, m>1: \Lambda_{V}^{s s d} /(S-$ equivalence $)=$ $\mathfrak{L}=\mathfrak{L}_{m}$, where $\Lambda_{V}^{s s d}$ is the semistable diagonal (see 4.2.4.a for the definition, cf. HM98]), and where $\mathfrak{L}$ is the exceptional fiber of the resolution of simple singularity;

$B P S$ states : certain constructible functions on our moduli spaces, see 4.2 .8 for details. 
4.1.3. Justification. If we consider the $D$-branes on $\widetilde{\mathbb{C}^{2} / \Gamma}$ then due to M. Kapranov and E. Vasserot [KV00:

$$
D^{b}\left(\operatorname{Coh}\left(\widetilde{\mathbb{C}^{2} / \Gamma}\right)\right) \simeq D^{b}(\operatorname{Rep}(Q))
$$

where $Q$ is the extended Dynkin diagram associated to the $\Gamma \subset S L(2, \mathbb{C})$ via the McKay correspondence, and $\operatorname{Rep}(Q)$ is the category of finite-dimensional double representations of $Q$ in the sense of [KV00, 3.4].

We are only interested in $D$-branes of charge $\alpha \in R_{+}$which are 0 -complexes on the right hand side, i.e. double representations of $Q$ rather than complexes of such representations. Such representations are identified with elements $x \in \mathbf{E}_{V}$, $\operatorname{dim} V=\alpha$ satisfying $m_{i}(x)=0$ for all $i \in I$ (see section 2.1.5 for the definition of $m_{i}$ ). The notion of stability becomes the usual GIT stability adapted to the quiver situation by King and Rudakov (see section 3). We choose a "non-degenerate" stability condition (cf. 4.2.6).

If $\operatorname{dim} V=\alpha \in R_{+}^{\mathrm{re}}$, then the moduli space is a point: $\mathcal{M}_{\zeta}(\alpha)=\mathrm{pt}_{\alpha}$.

If $\operatorname{dim} V=\delta$, where $\delta$ is the indivisible imaginary root, then the moduli space is the resolution of a simple singularity:

$$
\mathcal{M}_{\zeta}(\delta)=\widetilde{\mathbb{C}^{2} / \Gamma}
$$

In the two cases above one may replace the (middle) cohomology of the moduli spaces with the constructible functions on the exceptional fiber see [Nak94, 10.16]. Following this logic, we suggest the interpretation of BPS states as in the dictionary above and in 4.2 .8 below if $\operatorname{dim} V=m \delta, m>1$ where the situation is more complicated and a clear connection between physical and mathematical results was not available before, as far as we know.

4.1.4. Physics implies that the space of BPS states on $\widetilde{\mathbb{C}^{2} / \Gamma}$ of all possible charges should form a Lie algebra isomorphic to the plus part of the affine Lie algebra corresponding to $\Gamma$ via the McKay correspondence FM00. The main purpose of this section is to offer a conjectural, but mathematically rigorous, validation of this claim in 4.2.8.

4.2. The conjecture. In this section we consider quivers of affine ADE type, i.e. the underlying non-oriented graph $Q$ is an extended Dynkin graph of ADE type.

4.2.1. Let us fix an extending vertex $p$ of the graph $Q$. For every $\alpha=\operatorname{dim} V \operatorname{let}$ us fix the Nakajima's character: $\theta(V)=-1$, if $\operatorname{dim} V=k \in I^{\prime}=I-\{p\}$, and $\theta(V)=\sum_{i \in I^{\prime}} \operatorname{dim}_{\mathbb{C}}\left(V_{i}\right)$ if $\operatorname{dim} V=p \in I$ (cf. Nak98). (Semi)stable points in this section are considered with respect to this stability condition. If $V_{p}=0$ we can take a King's character $\Theta_{V, \Omega}$ (see 3.1.3) associated to any orientation that "flows to the extending vertex" i.e. the extending vertex is a sink and one can get to the extending vertex from any other point in the quiver going along the oriented edges. In the DE case there is only one such orientation (once the extending vertex is fixed). In the $A_{n}$ case there are $n$ such orientations.

4.2.2. For $\operatorname{dim} V=\alpha \in R_{+}^{\text {re }}$ we have (cf. CB01):

$$
\Lambda_{V}^{s} / G_{V}=\mathrm{pt}_{\alpha} .
$$

Let us denote by $\widetilde{E}_{\alpha}$ the function identically 1 on the point $\mathrm{pt}_{\alpha}, \widetilde{E}_{\alpha}\left(\mathrm{pt}_{\alpha}\right) \equiv 1$. 
4.2.3. For $\operatorname{dim} V=\delta=$ indivisible imaginary root we have (Kron89, Nak94):

$$
\Lambda_{V}^{s} / G_{V}=\mathfrak{L},
$$

where $\mathfrak{L}=\mathfrak{L}_{1}$ is the exceptional fiber of the resolution of simple singularity

$$
\mathfrak{L} \hookrightarrow \widetilde{\mathbb{C}^{2} / \Gamma} \rightarrow \mathbb{C}^{2} / \Gamma .
$$

Here $\Gamma$ is the finite subgroup of $S L(2, \mathbb{C})$ corresponding to the diagram $Q$ via the McKay correspondence.

It is well known that $\mathfrak{L}$ is a configuration of lines $\mathbb{P}^{1}$ which are its irreducible components and which may be indexed by the vertices $k \in I^{\prime}=I-\{p\}$. We will denote the $k^{\text {th }}$ irreducible component of $\mathfrak{L}$ by $Y_{k}, k \in I^{\prime}$. Let us denote the characteristic function of $Y_{k}$ by $\widetilde{E}_{k}(1), k \in I^{\prime}, \widetilde{E}_{k}(1)\left(Y_{k}\right) \equiv 1, \widetilde{E}_{k}(1)\left(\mathfrak{L}-Y_{k}\right) \equiv 0$.

4.2.4. Finally, for $\operatorname{dim} V=m \delta, m>1$ we introduce the semistable diagonal $\Lambda_{V}^{s s d} \subset \Lambda_{V}$ as follows (cf. [HM98]): $x$ is $S$-equivalent to the direct sum of $m$ isomorphic representations in $\Lambda_{\delta}^{s}$. Here $\Lambda_{\delta}^{s}=\Lambda_{V^{\prime}}^{s}, \operatorname{dim} V^{\prime}=\delta$. Formally:

$$
\Lambda_{V}^{s s d}=\left\{x \in \Lambda_{V} \mid x \stackrel{S}{\sim} x_{1} \oplus x_{2} \oplus \cdots \oplus x_{m}, x_{1} \simeq x_{2} \simeq \cdots \simeq x_{m}, x_{i} \in \Lambda_{\delta}^{s}\right\}
$$

It is clear that:

$$
\Lambda_{V}^{s s d} /(S \text {-equivalence })=\mathfrak{L}
$$

where $\mathfrak{L}=\mathfrak{L}_{m}$ is the same exceptional variety as above. Let us denote the characteristic function of the $k^{\text {th }}$ irreducible component $Y_{k}$ of $\mathfrak{L}=\mathfrak{L}_{m}$ by $\widetilde{E}_{k}(m), k \in I^{\prime}$, $\widetilde{E}_{k}(m)\left(Y_{k}\right) \equiv 1, \widetilde{E}_{k}(m)\left(\mathfrak{L}-Y_{k}\right) \equiv 0$.

4.2.5. We have defined the functions $E_{\alpha}^{*}$ on $\Lambda_{V}, \operatorname{dim} V=\alpha \in R_{+}^{\mathrm{re}}$, and the functions $E_{k}^{*}(m), k \in I^{\prime}=I-\{p\}$ on $\Lambda_{V}, \operatorname{dim} V=m \delta, m \geq 1$ in section 2.5. We need one more definition (cf. 2.5.0.b):

$$
\hat{E}_{k}^{*}(m):=\epsilon\left(\alpha_{k}, \beta_{k}\right)\left[\left.E_{\alpha_{k}}^{*}\right|_{\Lambda_{V_{1}}} ^{s},\left.E_{\beta_{k}}^{*}\right|_{\Lambda_{V_{2}}^{s}}\right]
$$

where $\operatorname{dim} V_{1}=\alpha_{k}=k \in I^{\prime} \subset R_{+}^{\mathrm{re}}, \operatorname{dim} V_{2}=\beta_{k}=\left(m \delta-\alpha_{k}\right) \in R_{+}^{\mathrm{re}}$ (here we treat elements of $I^{\prime}$ as simple roots), and the bracket is in the sense of 2.2.3.

Since the functions $E_{\alpha}^{*}, \hat{E}_{k}^{*}(m)$ are constant on $G_{V}$-orbits, we can consider them as functions on $\Lambda_{V} / G_{V}$. The equalities in the following conjecture are understood in this sense.

Conjecture. 4.2.5.a. Up to a sign, $\left.E_{\alpha}^{*}\right|_{\Lambda_{V}^{s}}=\widetilde{E}_{\alpha}, \operatorname{dim} V=\alpha \in R_{+}^{\mathrm{re}}$.

4.2.5.b. Up to a sign, $\left.\hat{E}_{k}^{*}(1)\right|_{\Lambda_{V}^{s}}=\widetilde{E}_{k}(1), \operatorname{dim} V=\delta$.

4.2.5.c. Let $\operatorname{dim} V=m \delta, m>1$. We conjecture that if $x^{\prime}, x^{\prime \prime} \in \Lambda^{\text {ssd }}$ are two elements in the same $S$-equivalence class and $\hat{E}_{k}^{*}(m)\left(x^{\prime}\right) \neq 0, \hat{E}_{k}^{*}(m)\left(x^{\prime \prime}\right) \neq$ 0 , then $\hat{E}_{k}^{*}(m)\left(x^{\prime}\right)=\hat{E}_{k}^{*}(m)\left(x^{\prime \prime}\right)$. Thus we can consider $\left.\hat{E}_{k}^{*}(m)\right|_{\Lambda_{V}^{s s d}}$ as a function on $\Lambda^{\text {ssd }} / S$-equivalence by setting for an $S$-equivalence class $X$ :

$$
\hat{E}_{k}^{*}(m)(X)= \begin{cases}\hat{E}_{k}^{*}(m)(x), & \text { if there exists } x \in X \text { with } \hat{E}_{k}^{*}(m)(x) \neq 0, \\ 0, & \text { otherwise. }\end{cases}
$$

4.2.5.d. $U p$ to a sign, $\left.\hat{E}_{k}^{*}(m)\right|_{\Lambda_{V}^{s s d}}=\widetilde{E}_{k}(m), \operatorname{dim} V=m \delta$.

The conjecture is verified in the $\hat{A}_{1}$ case. 
4.2.6. Remark. We would like to explain here that there is nothing special about the Nakajima's choice of character. If $\operatorname{dim} V=m \delta$ is an imaginary root, then the space of all characters defining stability may be naturally identified with a (classical) Cartan subalgebra $\mathfrak{h}=\mathbb{C}$-vector space spanned by $i \in I^{\prime}$. A character $\theta$ is called non-degenerate if it does not lie on a wall of a Weyl chamber. We expect the conjecture to be true for any non-degenerate character in the same Weyl chamber as the Nakajima's character (this is the fundamental chamber). Moreover, we could take a non-degenerate character in any other Weyl chamber, but that would change our choice of simple roots which we identify with the set $I^{\prime}=I-\{p\}$. One could perhaps reformulate our conjecture in this case modifying the functions $E_{k}^{*}(m)$ using the action of the Weyl group.

4.2.7. Remark. The functions $\widetilde{E}_{k}(m)$ parameterize the set $\operatorname{Irr} \mathfrak{L}=\operatorname{Irr} \mathfrak{L}_{m}$ of the irreducible components of the exceptional variety $\mathfrak{L}$. The components of $\mathfrak{L}$ are a basis in the space $H^{2}\left(\widetilde{\mathbb{C}^{2} / \Gamma}\right)$, see Nak94.

4.2.8. Denote the Lie algebra spanned by the functions $E_{\alpha}^{*}$ on $\Lambda_{V}, \operatorname{dim} V=\alpha \in$ $R_{+}^{\text {re }}$, and the functions $E_{k}^{*}(m) k \in I^{\prime}=I-\{p\}$ on $\Lambda_{V}, \operatorname{dim} V=m \delta, m \geq 1$ by $n^{*}(Q)$, cf. section 2.5 .

Let us denote the vector space spanned by the functions $\widetilde{E}_{\alpha}, \alpha \in R_{+}^{\text {re }}$, and the functions $\widetilde{E}_{k}(m)$ on $\mathfrak{L}=\mathfrak{L}_{m}=\Lambda_{m \delta}^{\text {ssd }} /(S-$ equivalence $)$ by $\mathfrak{n}^{\text {stable }}(Q)$. This is the vector space of constructible functions on moduli spaces of (semi)stable points in $\Lambda$, which are linear combinations of characteristic functions of irreducible components. The space $\mathfrak{n}^{\text {stable }}(Q)$ is our model of the space of BPS states at the orbifold $\mathbb{C}^{2} / \Gamma$, with $Q$ corresponding to $\Gamma$ via the McKay correspondence.

The conjecture, if true, would imply that the restriction from $\Lambda$ to $\Lambda^{s}$ (or $\Lambda^{s s d}$ ) gives us the based isomorphism of vector spaces

$$
\mathfrak{n}^{*}(Q) \stackrel{\simeq}{\longrightarrow} \mathfrak{n}^{\text {stable }}(Q)
$$

such that $E_{\alpha}^{*} \mapsto \widetilde{E}_{\alpha}$ and $E_{k}^{*}(m) \mapsto \hat{E}_{k}(m) \mapsto \widetilde{E}_{k}(m)$ for $\alpha \in R_{+}^{\mathrm{re}}, k \in I^{\prime}, m \geq 1$. This map equips the space of BPS states $\mathfrak{n}^{\text {stable }}(Q)$ with the structure of a Lie algebra isomorphic to the plus part of the affine Lie algebra corresponding to $Q$.

On the other hand the above isomorphism provides the plus part of the affine Lie algebra corresponding to $Q$, with a basis $\left\{\widetilde{E}_{\alpha}, \alpha \in R_{+}^{\text {re }} ; \widetilde{E}_{k}(m), k \in I^{\prime}, m \in \mathbb{Z}_{>0}\right\}$ indexed by irreducible components of algebraic varieties $\mathrm{pt}_{\alpha}=\Lambda_{\alpha}^{s} / G_{\alpha}$, and $\mathfrak{L}_{m}=$ $\Lambda_{m \delta}^{s s d} /(S-$ equivalence $), m \in \mathbb{Z}_{>0}$.

\section{Remarks on Semicanonical basis for Simple Lie algebras}

5.1. Semicanonical basis. This subsection is lifted from [Lus00, 2.4-5]. Recall the setup of 2.3. Let $V=\oplus_{i \in I} V_{i}$ be a $I$-graded vector space. If $Y \in \operatorname{Irr} \Lambda_{V}$ is an irreducible component of $\Lambda_{V}$ and $f \in \mathcal{L}_{\mathbb{Z}}(V)$ then there is a unique $c \in \mathbb{Z}$ such that $f^{-1}(c) \cap Y$ contains an open dense subset of $Y$. Note that $f \mapsto c$ is a linear function $\rho_{Y}: \mathcal{L}_{\mathbb{Z}}(V) \rightarrow \mathbb{Z}$.

Lemma. Let $Y \in \operatorname{Irr} \Lambda_{V}$. There exists $f=e_{Y} \in \psi_{V}\left(U_{\mathbb{Z}, V}^{+}\right)$such that $\rho_{Y}(f)=1$ and $\rho_{Y^{\prime}}(f)=0$ for any $Y^{\prime} \in \operatorname{Irr} \Lambda_{V}-\{Y\}$.

Lusztig proves that the functions $e_{Y}, Y \in \operatorname{Irr} \Lambda_{V}$ form a basis in $\psi_{V}\left(U_{V}^{+}\right)$, and therefore, the collection of $e_{Y}, Y \in \operatorname{Irr} \Lambda_{V}$ for all possible dimensions of $V$ forms a 
basis in the algebra $\psi\left(U^{+}\right)=\mathcal{L}_{0}$ isomorphic to $U^{+}$. This basis is called semicanonical.

In the remainder of this section we consider quivers of finite ADE type.

5.1.1. Since our quiver is of finite ADE type we know [Lus91, 14.2] that the irreducible components of $\Lambda_{V}$ are the closures of conormal bundles of the various $G_{V}$-orbits in $\mathbf{E}_{V, \Omega}$, where $\Omega$ is some orientation. Such $G_{V}$-orbits are indexed by the decomposition of $(V, x), x \in \mathbf{E}_{V, \Omega}$ into a direct sum of indecomposable submodules $(V, x)=\left(V_{1}, x_{1}\right) \oplus \cdots \oplus\left(V_{l}, x_{l}\right)$ with $x_{i} \in \mathbf{E}_{V_{i}, \Omega}^{\text {ind }}$. (The decompositions are considered up to the order of the summands.) Thus, there is a one-to one correspondence between $\operatorname{Irr} \Lambda_{V}$ and the decompositions $\operatorname{dim} V=\operatorname{dim} V_{1}+\cdots+\operatorname{dim} V_{l}$ of $\operatorname{dim} V \in \mathbb{Z}[I]$ into a sum of positive roots $\operatorname{dim} V_{i} \in R_{+}$. (Recall that $\mathbf{E}_{V, \Omega}^{\text {ind }} \neq \emptyset$ if and only if $\operatorname{dim} V \in R_{+}$.) Thus the elements of the semicanonical basis may be indexed by decompositions of $\operatorname{dim} V$ into a sum of positive roots.

5.2. Open problem. Recall that we have defined the functions $E_{\alpha}^{*}$ on $\Lambda_{V}, \operatorname{dim} V=$ $\alpha \in R_{+}$in 2.4. By construction these functions are defined canonically up to a sign which depends on the choice of the cocycle $\epsilon$. Since $E_{\alpha}^{*} \in \psi_{V}\left(U_{\mathbb{Z}, \operatorname{dim} V}^{+}\right)$, we can decompose $E_{\alpha}^{*}$ with respect to the semicanonical basis:

$$
E_{\alpha}^{*}=\sum_{Y \in \operatorname{Irr} \Lambda_{V}} c_{Y} e_{Y}
$$

The problem is to calculate the integer coefficients $c_{Y}$ for all irreducible components $Y$ of $\Lambda_{V}$. These coefficients are independent of any choices up to simultaneous multiplication of all of them by -1 . Suppose that an irreducible component $Y \in$ $\operatorname{Irr} \Lambda_{V}, \operatorname{dim} V=\alpha$ corresponds to a decomposition

$$
\alpha=\alpha_{1}+\cdots+\alpha_{l}
$$

of $\alpha$ into a sum of positive roots $\alpha_{1}, \ldots, \alpha_{l}$. Then the formula 5.2.0.a may be regarded as an assignment $\left\{\alpha=\alpha_{1}+\cdots+\alpha_{l}\right\} \mapsto c_{Y}$ of the integer $c_{Y}$ to every decomposition 5.2.0.b. It would be very interesting to describe this assignment in terms of combinatorics of the root system without appealing to the semicanonical basis. We obtain such a description in the $A_{n}$ case using the Weyl group $W=S_{n+1}$. We also succeed in calculating $c_{Y}$ when $Y=\mathbf{E}_{V, \Omega}$ are irreducible components "arising from orientations", and we calculate the examples of $D_{4}$ and $D_{5}$.

\subsection{Irreducible components arising from orientations.}

5.3.1. Let us define the subvariety $O_{V} \subseteq \Lambda_{V}$ as follows:

$$
O_{V}=\bigcup_{\Omega} \mathbf{E}_{V, \Omega}
$$

where the union is taken over all orientations $\Omega$ of our quiver. Since each $Y=\mathbf{E}_{V, \Omega}$ is an irreducible component of $\Lambda_{V}$ (we will call such $Y$ irreducible components arising from orientations), we have $\operatorname{Irr} O_{V} \subseteq \operatorname{Irr} \Lambda_{V}$. We will split the sum 5.2.0.a into two parts:

$$
E_{\alpha}^{*}=E_{\alpha}^{\prime}+E_{\alpha}^{\prime \prime}
$$

where

$$
E_{\alpha}^{\prime}=\sum_{Y \in \operatorname{Irr} O_{V}} c_{Y} e_{Y} \quad \text { and } \quad E_{\alpha}^{\prime \prime}=\sum_{Y \in \operatorname{Irr} \Lambda_{V}-\operatorname{Irr} O_{V}} c_{Y} e_{Y}
$$


5.3.2. If a root $\alpha \in R_{+}$is not simple, represent it as a sum of simple roots $\alpha_{k_{1}}, \alpha_{k_{2}}, \ldots, \alpha_{k_{h}}, k_{l} \in I$, where $h=$ ht $\alpha$

$$
\alpha=\alpha_{k_{1}}+\alpha_{k_{2}}+\cdots+\alpha_{k_{h}}
$$

in such a way that $\alpha_{k_{1}}+\alpha_{k_{2}}+\cdots+\alpha_{k_{j}}$ is a root for $1 \leq j \leq h$.

It follows from our construction and definitions that up to a sign

$$
E_{\alpha}^{*}=\left[\ldots\left[E_{\alpha_{k_{1}}}^{*}, E_{\alpha_{k_{2}}}^{*}\right] \ldots E_{\alpha_{k_{h}}}^{*}\right]
$$

Now we can calculate the coefficients of $E_{\alpha}^{\prime}$ (cf. section 3 for a similar discussion).

Proposition. Up to a sign,

$$
E_{\alpha}^{\prime}=\sum_{Y=\mathbf{E}_{V, \Omega} \in \operatorname{Irr} O_{V}}\left(\prod_{i<j} \epsilon_{\Omega}\left(\alpha_{k_{i}}, \alpha_{k_{j}}\right)\right) e_{Y},
$$

where $\epsilon_{\Omega}$ is the Frenkel-Kac cocycle corresponding to $\Omega$, [FMV01, 1.3.4]. This presentation does not depend on the decomposition 5.3.2.d.

Proof. Let us fix some orientation $\Omega$, and let us assume that $Y=\mathbf{E}_{V, \Omega}$. According to Lusztig Lus92, 12.13] we have a homomorphism of algebras $\mathcal{L}_{0} \rightarrow \mathcal{L}_{0, \Omega}$ induced by the restriction on functions from $\Lambda_{V}$ to $\mathbf{E}_{V, \Omega}$ (see 2.2 .3 for the definitions of $\mathcal{L}_{0}$ and $\left.\mathcal{L}_{0, \Omega}\right)$. Then

$$
\left.E_{\alpha}^{*}\right|_{Y}=\left.E_{\alpha}^{\prime}\right|_{Y}=\left[\ldots\left[E_{k_{1}}, E_{k_{2}}\right]_{\Omega} \ldots E_{k_{h}}\right]_{\Omega}=\prod_{i<j} \epsilon_{\Omega}\left(\alpha_{k_{i}}, \alpha_{k_{j}}\right) E_{\alpha}
$$

where $E_{\alpha}$ is defined by 3.3.2.a, and the last equality follows from [FMV01, 4.3.4.b]. Now it is clear that $\rho_{Y}\left(E_{\alpha}^{*}\right)=\rho_{Y}\left(\left.E_{\alpha}^{*}\right|_{Y}\right)$. Then

$$
c_{Y}=\rho_{Y}\left(E_{\alpha}^{*}\right)=\rho_{Y}\left(\left.E_{\alpha}^{*}\right|_{Y}\right)=\prod_{i<j} \epsilon_{\Omega}\left(\alpha_{k_{i}}, \alpha_{k_{j}}\right) \rho_{Y}\left(E_{\alpha, \Omega}\right)=\prod_{i<j} \epsilon_{\Omega}\left(\alpha_{k_{i}}, \alpha_{k_{j}}\right) .
$$

Notice that due to the formula 5.3.2.c we know explicitly $\left.E_{\alpha}^{*}\right|_{O}$ i.e., the value $E_{\alpha}^{*}(x)$ at any point $x \in O \subseteq \Lambda$.

5.3.3. Let $Q$ be the Dynkin graph of type $A_{n}$. In this case it is clear that for $V \in \mathcal{V}, \operatorname{dim} V=\alpha \in R_{+}$any irreducible component of $\Lambda_{V}$ is of the form $\mathbf{E}_{V, \Omega}$ for some orientation $\Omega$. Thus Proposition 5.3.2 gives a complete answer to our question 5.2 in this case.

\subsection{Conjugacy classes of the Weyl group.}

5.4.1. Recall the isomorphism $\psi: \mathfrak{n} \rightarrow \mathfrak{n}^{*}$ (see 2.3.2), and let us take $\tilde{e}_{\alpha} \in \mathfrak{n}^{\epsilon} \simeq \mathfrak{n}$ such that $\psi\left(\tilde{e}_{\alpha}\right)=E_{\alpha}^{*}, \alpha \in R_{+}$, as in 2.4. Let us fix $Y \in \operatorname{Irr} \Lambda_{V}$ and let $\alpha=$ $\beta_{1}+\cdots+\beta_{l}$ be the corresponding decomposition of $\alpha$ into the sum of positive roots. Now consider the element $\tilde{e}_{\beta_{1}}+\cdots+\tilde{e}_{\beta_{l}} \in \mathfrak{n}^{\epsilon} \simeq \mathfrak{n}$, and consider its adjoint orbit. In such a way we obtain a map from $\operatorname{Irr} \Lambda_{V}$ to the set $\mathcal{N}$ of nilpotent orbits of the Lie algebra corresponding to the Dynkin diagram $Q$. Moreover, following Kazhdan-Lusztig [KL88] and Spaltenstein [Spal88], we can construct a map from $\mathcal{N}$ to the set $\mathrm{Cl}(W)$ of conjugacy classes of the Weyl group $W$. Thus we obtain a map cl : $\operatorname{Irr} \Lambda_{V} \rightarrow \mathrm{Cl}(W)$ for any $V \in \mathcal{V}$. We will use this map in the following subsection. 
5.4.2. Example: $A_{n}$.

Proposition. Up to a sign,

$$
E_{\alpha}^{*}=\sum_{Y \in \operatorname{Irr} \Lambda_{V}} \operatorname{sgn}(\operatorname{cl} Y) e_{Y},
$$

where $\operatorname{sgn}(\mathrm{cl} Y)$ is the value of the one-dimensional sign character of $W=S_{n+1}$ on the conjugacy class $\mathrm{cl} Y$.

Proof. The proof is straightforward and is left to the reader.

5.4.3. Open question. We have the map cl $: \operatorname{Irr} \Lambda_{V} \rightarrow \mathrm{Cl}(W)$ for all ADE quivers, see 5.4.1. Looking at the $A_{n}$ case (Proposition 5.4.2), one may ask if in the DE case the coefficients $c_{Y}$ are also governed by characters of the Weyl group. Unfortunately, the authors' naive attempt to replicate the $A_{n}$ result fails already in the $D_{4}$ case (however the explicit calculation for the maximal root of $D_{4}$ is given below).

\subsection{Example: $D_{4}$.}

5.5.1. We index the vertices of the Dynkin diagram as follows:

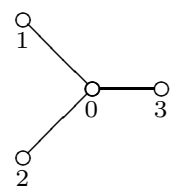

The simple roots are denoted by $\alpha_{0}, \alpha_{1}, \alpha_{2}, \alpha_{3}$. Let $\alpha=2 \alpha_{0}+\alpha_{1}+\alpha_{2}+\alpha_{3}$ be the maximal root. Indexing the irreducible components of $\Lambda_{V}, \operatorname{dim} V=\alpha$ by the decompositions of $\alpha$ we obtain by a straighforward computation using the properties of the semicanonical basis:

$$
\begin{aligned}
E_{\alpha}^{*} & =E_{\alpha}^{\prime}+E_{\alpha}^{\prime \prime} \\
& =E_{\alpha}^{\prime}-e_{\left(\alpha_{0}+\alpha_{1}\right)+\left(\alpha_{0}+\alpha_{2}+\alpha_{3}\right)}-e_{\left(\alpha_{0}+\alpha_{2}\right)+\left(\alpha_{0}+\alpha_{1}+\alpha_{3}\right)}-e_{\left(\alpha_{0}+\alpha_{3}\right)+\left(\alpha_{0}+\alpha_{1}+\alpha_{2}\right)} \\
& -e_{\alpha_{0}+\alpha_{1}+\left(\alpha_{0}+\alpha_{2}+\alpha_{3}\right)}-e_{\alpha_{0}+\alpha_{2}+\left(\alpha_{0}+\alpha_{1}+\alpha_{3}\right)}-e_{\alpha_{0}+\alpha_{3}+\left(\alpha_{0}+\alpha_{1}+\alpha_{2}\right)} \\
& +2 e_{\alpha_{0}+\left(\alpha_{0}+\alpha_{1}+\alpha_{2}+\alpha_{3}\right) .}
\end{aligned}
$$

\subsection{Example: $D_{5}$.}

5.6.1. We index the vertices of the Dynkin diagram as follows:

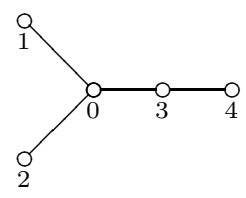

The simple roots are denoted by $\alpha_{0}, \alpha_{1}, \alpha_{2}, \alpha_{3}, \alpha_{4}$. Let $\alpha=2 \alpha_{0}+\alpha_{1}+\alpha_{2}+$ $2 \alpha_{3}+\alpha_{4}$ be the maximal root. There are 55 root partitions of $\alpha$, e.g. $\alpha=$ $\alpha_{0}+\alpha_{3}+\left(\alpha_{0}+\alpha_{1}+\alpha_{2}+\alpha_{3}+\alpha_{4}\right)$. Indexing the irreducible components of $\Lambda_{V}$, 
$\operatorname{dim} V=\alpha$ by the decompositions of $\alpha$ we obtain by a tedious but straightforward computation using the properties of the semicanonical basis:

$$
\begin{aligned}
& E_{\alpha}^{*}=\quad E_{\alpha}^{\prime}+E_{\alpha}^{\prime \prime}=E_{\alpha}^{\prime}+ \\
& +e_{\left(2 \alpha_{0}+\alpha_{1}+\alpha_{2}+\alpha_{3}\right)+\left(\alpha_{3}+\alpha_{4}\right)+e_{\alpha_{2}}+\left(\alpha_{0}+\alpha_{1}\right)+\left(\alpha_{0}+\alpha_{3}\right)+\left(\alpha_{3}+\alpha_{4}\right)} \\
& +e_{\alpha_{1}+\left(\alpha_{0}+\alpha_{2}\right)+\left(\alpha_{0}+\alpha_{3}\right)+\left(\alpha_{3}+\alpha_{4}\right)}+e_{\alpha_{1}}+\alpha_{2}+\left(\alpha_{0}+\alpha_{3}\right)+\alpha_{0}+\left(\alpha_{3}+\alpha_{4}\right) \\
& +e_{\left(2 \alpha_{0}+\alpha_{1}+\alpha_{2}+\alpha_{3}\right)+\alpha_{3}+\alpha_{4}+e_{\alpha_{2}}+\left(\alpha_{0}+\alpha_{1}\right)+\alpha_{3}+\alpha_{4}+\left(\alpha_{0}+\alpha_{3}\right)} \\
& +e_{\alpha_{1}+\left(\alpha_{0}+\alpha_{2}\right)+\left(\alpha_{0}+\alpha_{3}\right)+\alpha_{3}+\alpha_{4}}+e_{\left(\alpha_{0}+\alpha_{3}\right)+\alpha_{0}+\alpha_{2}+\alpha_{3}+\alpha_{4}}
\end{aligned}
$$

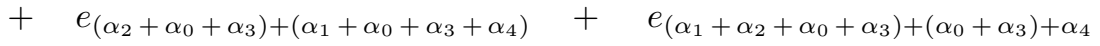

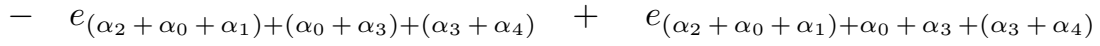

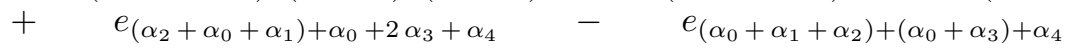

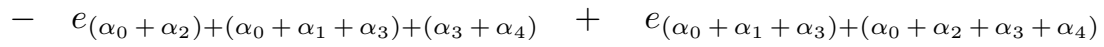

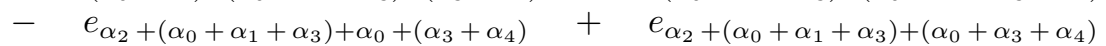

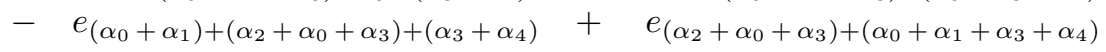

$$
\begin{aligned}
& -e_{\alpha_{0}+\alpha_{1}+\left(\alpha_{2}+\alpha_{0}+\alpha_{3}\right)+\left(\alpha_{3}+\alpha_{4}\right)}+e_{\alpha_{1}+\left(\alpha_{2}+\alpha_{0}+\alpha_{3}\right)+\left(\alpha_{0}+\alpha_{3}+\alpha_{4}\right)} \\
& +e_{\left(\alpha_{0}+\alpha_{2}\right)+\alpha_{3}+\left(\alpha_{0}+\alpha_{1}+\alpha_{3}+\alpha_{4}\right)}-e_{\left(\alpha_{0}+\alpha_{2}\right)+\alpha_{3}+\left(\alpha_{0}+\alpha_{1}+\alpha_{3}\right)+\alpha_{4}} \\
& +e_{\alpha_{0}+\alpha_{2}+\alpha_{3}+\left(\alpha_{0}+\alpha_{1}+\alpha_{3}+\alpha_{4}\right)}-e_{\alpha_{0}+\alpha_{2}+\alpha_{3}+\left(\alpha_{0}+\alpha_{1}+\alpha_{3}\right)+\alpha_{4}}
\end{aligned}
$$

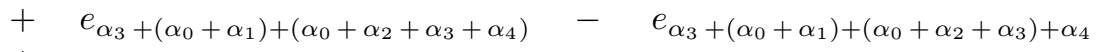

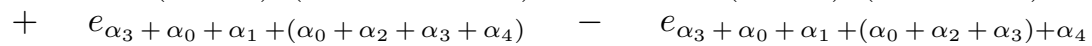

$$
\begin{aligned}
& +2 e_{\alpha_{0}+\left(\alpha_{3}+\alpha_{4}\right)+\left(\alpha_{0}+\alpha_{1}+\alpha_{2}+\alpha_{3}\right)}-2 e_{\left(\alpha_{0}+\alpha_{3}+\alpha_{4}\right)+\left(\alpha_{0}+\alpha_{1}+\alpha_{2}+\alpha_{3}\right)}
\end{aligned}
$$

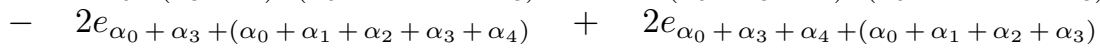

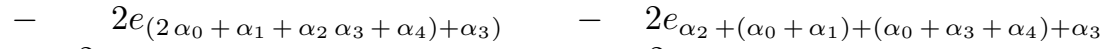

$$
\begin{aligned}
& -2 e_{\alpha_{1}}+\left(\alpha_{0}+\alpha_{2}\right)+\left(\alpha_{0}+\alpha_{3}+\alpha_{4}\right)+\alpha_{3}-2 e_{\left(\alpha_{0}+\alpha_{3}+\alpha_{4}\right)+\alpha_{0}+\alpha_{1}+\alpha_{2}+\alpha_{3}} \\
& +2 e_{\left(\alpha_{0}+\alpha_{3}+\alpha_{4}\right)+\left(\alpha_{0}+\alpha_{1}+\alpha_{2}\right)+\alpha_{3}}
\end{aligned}
$$

5.6.2. While in the $D_{5}$ case the coefficients in the decomposition for the maximal root are $\pm 1, \pm 2$, in the $D_{6}$ case the coefficients are $\pm 1, \pm 2, \pm 4$. We expect that in the $D_{n}$ case all the coefficients are the powers of 2 .

5.7. More open questions. The questions of decomposing "canonically" defined basis elements of affine Lie algebra (such as $E_{\alpha}^{*}, E_{k}^{*}(n)$, see 2.5) with respect to semicanonical basis make sense for any affine Lie algebra of type ADE. It would be very interesting to develop a purely combinatorial approach to these questions in the affine case as well.

\section{REFERENCES}

[CB01] W. Crawley-Boevey, Geometry of the moment map for representations of quivers, Compositio Math. 126 (2001), no. 3, 257-293.

[DFR00] M. Douglas. B. Fiol, and C. Römelsberger, Stability and BPS branes, preprint 2000, hep-th/0002037.

[FM00] B. Fiol and M. Mariño, BPS states and algebras from quivers, J. High Energy Phys. 2000, no. 7, Paper 31, 40 pp.

[FK81] I. Frenkel and V. Kac, Basic representations of affine Lie algebras and dual resonance models, Invent. Math. 62 (1980/81), no. 1, 23-66.

[FMV01] I. Frenkel, A. Malkin, and M. Vybornov, Affine Lie Algebras and Tame Quivers, Selecta Math. (N.S.) 7 (2001), no. 1, 1-56.

[Gab72] P. Gabriel, Unzerlegbare Darstellungen. I, Manuscripta Math. 6 (1972), 71-103; correction, ibid. 6 (1972), 309.

[HM98] J. Harvey and G. Moore, On the algebras of BPS states, Comm. Math. Phys. 197 (1998), no. 3, 489-519.

[KV00] M. Kapranov and E. Vasserot Kleinian singularities, derived categories and Hall algebras, Math. Ann. 316 (2000), no. 3, 565-576. 
[KS97] M. Kashiwara and Y. Saito, Geometric Construction of Crystal bases, Duke Math. J. 89 (1997), no. 1, 9-36.

[KL88] D. Kazhdan and G. Lusztig, Fixed point varieties on affine flag manifolds, Israel J. Math. 62 (1988), no. 2, 129-168.

[King94] A. King, Moduli of representations of finite dimensional algebras, Quart. J. Math. Oxford (2), 45, (1994), 515-530.

[Kron89] P. Kronheimer, The construction of ALE spaces as hyper-Kähler quotients, J. Differential Geom. 29 (1989), no. 3, 665-683.

[Lus90] G. Lusztig, Canonocal bases arising from quantized enveloping algebras, J. Amer. Math. Soc. 3 (1990), no. 2, 447-498.

[Lus91] G. Lusztig, Quivers, perverse sheaves, and quantized enveloping algebras, J. Amer. Math. Soc. 4 (1991), no. 2, 365-421.

[Lus92] G. Lusztig, Affine quivers and canonical bases, Inst. Hautes Études Sci. Publ. Math. (1992), no. $76,111-163$.

[Lus00] G. Lusztig, Semicanonical bases arising from enveloping algebras, Adv. Math. 151 (2000), no. 2, 129-139.

[Nak94] H. Nakajima, Instantons on ALE spaces, quiver varieties, and Kac-Moody algebras, Duke Math. J. 76 (1994), no. 2, 365-416.

[Nak98] H. Nakajima, Quiver varieties and Kac-Moody algebras, Duke Math. J. 91 (1998), no. $3,515-560$.

[Rei02] M. Reineke, The Harder-Narasimhan system in quantum groups and cohomology of quiver moduli, preprint 2002, math.QA/0204059.

[Rin90] C. Ringel, Hall poynomials for the representation-finite hereditary algebras, Adv. Math. 84 (1990), no. 2, 137-178.

[Rud97] A. Rudakov, Stability for an abelian category J. Algebra 197 (1997), no. 1, 231-245.

[Scho92] A. Schofield, General representations of quivers, Proc. London Math. Soc. (3) 65 (1992), 46-64.

[Spal88] N. Spaltenstein, Polynomials over local fields, nilpotent orbits and conjugacy classes in Weyl groups Orbites unipotentes et représentations, I. Astérisque No. 168 (1988), 10-11, 191-217.

Department of Mathematics, Yale University, 10 Hillhouse Ave, New Haven, CT 06520

E-mail address: frenkel@math.yale.edu

Department of Mathematics, Mit, 77 Massachusetts Avenue, Cambridge, Ma 02139

E-mail address: malkin@math.mit.edu

Department of Mathematics, University of Massachusetts, Lederle Graduate ReSEArCh Tower, Amherst, MA 01003

E-mail address: vybornov@math.umass.edu 